\title{
Photometric Evidence for Mixing and Diffusion in GC Stars
}

\author{
Frank Grundahl \\ Institute of Physics and Astronomy, Aarhus University, Ny Munkegade, \\ 8000 Aarhus C, Denmark
}

\begin{abstract}
Based on Strömgren uvby CCD photometry, and UVES spectroscopy we present evidence that possibly all globular clusters show large variations in their abundances of nitrogen, aluminium and sodium, which are not only due to internal mixing processes. Furthermore the evidence for radiative levitation among hot horizontal branch stars is briefly discussed.
\end{abstract}

\section{Introduction}

In an extremely stimulating paper, Sweigart (1997), proposed that deep mixing in globular cluster red giant branch (RGB) stars could have significant effects on the color and morphology of the horizontal branch. The suggestion was based on the fact that if the observed enhancements of $\mathrm{Al}$ were due to mixing then $\mathrm{He}$ produced in the $\mathrm{H}$ burning shell would also be mixed to the surface. In order to test to what extent deep mixing occurs in such RGB stars it is necessary to gather information on the observed abundance patterns in fainter (and presumably nonmixed) RGB stars. In this contribution we will discuss some of the information available on this subject based on uvby photometry. We shall also briefly discuss the presence of radiative levitation in hot horizontal branch stars.

\section{2. $\quad c_{1}$ scatter in RGB stars}

Grundahl, VandenBerg \& Andersen (1998) showed that the cluster M13 had a large star-to-star spread in the measured $c_{1}$ index among RGB stars, including sub-giant stars. Subsequent observations for many more clusters (22 in total) has shown that ALL clusters have such $c_{1}$ scatter, irrespective of their mass, galactocentric distance, age or any other parameters (Grundahl et al., 2000, in preparation). In Fig. 1 examples of such scatter are presented for 4 clusters covering the range -0.7 to -2.3 in $[\mathrm{Fe} / \mathrm{H}]$. It has been speculated (e.g. Grundahl et al. 1999, Grundahl 1999) that the cause for the $c_{1}$ scatter is large star-to-star variations in the NH (3360 $\AA$ ) strength and thus $\mathrm{N}$ abundance. This feature is included in the passband for the $u$ filter. The data for the plot were obtained with the Nordic Optical Telescope and the Danish $1.54 \mathrm{~m}$ on La Silla.

In the panels of Fig. 1 the vertical line marks the approximate location of the RGB bump where mixing is expected to start (Sweigart \& Mengel 1979, Gratton et al. 2000). Note, that for NGC 288, M13 and M92 there is a narrowing 


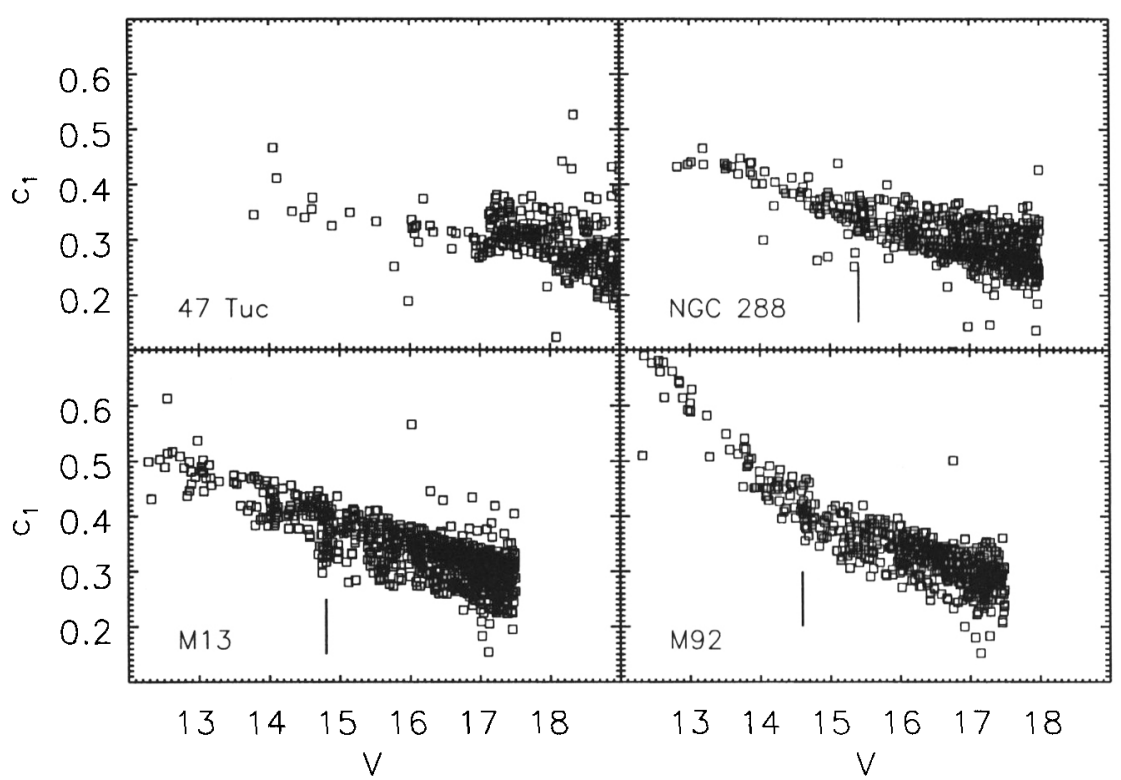

Figure 1. Plot showing the observed $\left(V, c_{1}\right)$ for four of our clusters. In 47Tuc, the scatter is primarily detected on the main-sequence since we only have a small RGB sample. In NGC 288 the RGB bump is not clearly detected, so the vertical line only marks the "expected" location of this feature.

of the $c_{1}$ as the luminosity increases from the bump towards the RGB tip. In the case of M92 there also seems to be a change of slope in the $\left(V, c_{1}\right)$ relation. We emphasize that these trends are not just present in these three clusters, but also in all of the remaining clusters on our observed list. The most straightforward explanation for this is (given that NH is the source of the $c_{1}$ variations) that the stars with low $c_{1}$ (weak $\mathrm{NH}$ band) experience a much larger relative change in $\mathrm{N}$ abundance than the high $c_{1}$ stars, when the surface $\mathrm{N}$ abundance is increased due to the onset of mixing. The results of Gratton et al. (2000) strongly suggest that there is no evidence for deep mixing below the bump level. If this holds true then we conclude based on our observed sample of 22 clusters that they all have strongly varying $\mathrm{N}$ abundances - thus we should not be surprised if other elements exhibit similar variations.

In fact, in an observing programme with Briley, Feltzing and Nissen we have just prior to the writing of this contribution obtained high resolution spectra ( $R \sim 60000, \mathrm{~S} / \mathrm{N} \sim 100$ ) with VLT/UVES for 21 stars close to the RGB bump in NGC 6752. A first inspection of these spectra indicates that i) the $c_{1}$ scatter correlates with the $3360 \AA \mathrm{NH}$ strength; ii) $\mathrm{Li}$ is present in all stars fainter but not brighter than the bump; iii) $\mathrm{Al}$ and $\mathrm{Na}$ varies strongly in stars both brighter at and fainter than the bump. These results suggest that before we can properly asses the role of mixing in the brightest cluster stars it is necessary to properly evaluate the extent of "primordial" variations in the abundances of the most important trace elements such as e.g. $\mathrm{Na}, \mathrm{Mg}, \mathrm{O}, \mathrm{Al}$. 


\section{Radiative levitation in hot horizontal branch stars}

In Grundahl et al. (1999) it is shown that in a $(u-y, u)$ color-magnitude diagram horizontal branch stars hotter than $\sim 11500 \mathrm{~K}$ appear too luminous or too hot as compared to canonical HB models. Based on several lines of evidence it is concluded by Grundahl et al. that the cause for this effect, for M13 is that the surface abundance of heavy elements such as eg. Fe is very strongly enhanced due to the effects of radiative levitation. Simultaneously and independently, Behr et al. (1999) found that the abundances of $\mathrm{Ph}, \mathrm{Fe}, \ldots .$. were enhanced by very large factors - thus confirming the levitation hypothesis. Moehler et al. (2000) have made further investigations of medium resolution spectra in hot $\mathrm{HB}$ stars in NGC6752. They also find evidence for the presence of levitation, but conclude that levitation alone is not sufficient to account for the $u$-jump (and the associated $\log g$ jump). For more details on the aspects of levitation, the reader is referred to the papers by Grundahl et al. (1999), Behr et al. (1999, 2000), Moehler et al. (2000) and Sweigart (this volume).

Acknowledgments. I wish to thank Carlsbergfondet for generous support which made this research possible and also M. Briley, M. Catelan, J.E. Hesser, W. Landsman and A. Sweigart for stimulating discussions and helpful advice on the topics discussed here. Peter Stetson is thanked for allowing me to use his excellent photometry software.

\section{References}

Behr, B.B., Cohen, J.G., McCarthy, J.K. \& Djorgowski, S.G., 1999, ApJ, 517, L135

Behr, B.B., et al. 2000, ApJ, 528, 849

Gratton, R.G., Sneden, C., Carretta, E., \& Bragaglia, A., 2000, A\&A, 354, 169

Grundahl, F., VandenBerg, D.A., \& Andersen, M.I, 1998, ApJ, 500, L179

Grundahl, F., Catelan, M., Landsman, W., Stetson, P.B., \& Andersen, M.I, 1999, ApJ, 524, 242

Grundahl, F., 1999, in Spectrophotometric Dating of Stars and Galaxies, ASP Conf. Series, Vol. 192, Eds. I. Hubeny, S.R. Heap and R.H. Cornett (San Francisco: ASP), 223

Moehler, S., Sweigart, A.V., Landsman, W.B., \& Heber, U., A\&A, 360, 120

Sweigart, A.V. \& Mengel, J.G., 1979, ApJ, 229, 624

Sweigart, A.V., 1997, in The Third Conference on Faint Blue Stars, ed. A.G.D. Phillip, J. Liebert \& R.A. Saffer (Schenectady: Davis), 3 